\title{
Etched chalcogenide fibers for near-field infrared scanning microscopy
}

\author{
M. A. Unger, ${ }^{\text {a) }}$ D. A. Kossakovski, R. Kongovi, J. L. Beauchamp, and J. D. Baldeschwieler \\ Division of Chemistry 127-72, California Institute of Technology, Pasadena, California 91125
}

D. V. Palanker

Stanford University, H.E.P.L., Free Electron Laser Center, Stanford, California 94305-4085

(Received 12 December 1997; accepted for publication 8 April 1998)

Typical infrared transmitting fibers comprise a chalcogenide core surrounded by a sulfur-selenide cladding, which is in turn coated with a polymer such as polyamide. For use in a near-field scanning infrared microscope (NSIM), such infrared-transmitting fibers must be tapered to a sharp point. Sharper points allow smaller apertures, which allow higher resolution. The light throughput of the probe depends on the length of the taper region: the longer the taper length, the further the infrared radiation must propagate through a waveguide smaller than its wavelength. Thus, shorter taper lengths should give higher light throughput. We describe a method for etching chalcogenide fibers to submicron points by simple chemical means. Methods are described for removal of the polyamide coating, stripping of the SSe cladding surrounding the core, and etching the chalcogenide fiber core to a sharp point. Removal of the polyamide coating is most easily accomplished by dissolution in 4-Chloro-1-butanol. The SSe cladding is removed by soaking the fiber in $0.1 \mathrm{M} \mathrm{NaOH}$ overnight. The chalcogenide core is tapered to a sharp point by immersion in a two-phase etching system, where the top phase is an inert organic solvent, and the bottom phase is a strong oxidant. Fibers both with and without cladding have been tapered. The resulting fibers have a taper length on the order of the core diameter, and terminate with a submicron end radius of curvature. The potential for use in a NSIM, as well as other uses, is discussed. () 1998 American Institute of Physics.

[S0034-6748(98)00907-1]

\section{INTRODUCTION}

Near-field scanning optical microscopy (NSOM) allows optical microscopy ${ }^{1-5}$ and spectroscopy ${ }^{4-6}$ with resolution on the order of $10-100 \mathrm{~nm}$, which is far smaller than the far-field diffraction limit $(\lambda / 2)$. Part of the price for achieving this resolution is the necessity of constructing a probe with an aperture of the size of the desired resolution. As with all scanned probe techniques, the power of the technique depends in large part on the quality of the probe.

Typical NSOM instruments use a pulled optical fiber, coated with metal except for an aperture at the apex, for a probe. They are therefore limited to the wavelength range transmitted by the fiber. With glass $\left(\mathrm{SiO}_{2}\right)$ fibers, this is typically ${ }^{7} 375-850 \mathrm{~nm}$. The throughput (measured in the far field) is typically ${ }^{8} 10^{-5}-10^{-6}$.

Near-field scanning infrared microscopy (NSIM) is an obvious extrapolation of the NSOM technique. In principle, NSIM has a definite advantage over NSOM: In contrast to the visible region, almost every molecule has an absorption in the IR region. Furthermore, IR absorption bands give direct information about the presence and nature of molecular bonds in the observed region.

High spatial resolution Raman microscopy is another way to extract molecular specific information from a sample. It is known that Raman and IR absorption spectroscopy provide somewhat complementary information due to the nature of IR radiation interaction with the matter. However, NSIM

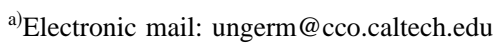

with a tapered fiber probe provides an advantage over Raman imaging: The probe is maintained at constant distance from the sample while scanning which provides topographical information in addition to IR absorption data. As in NSOM, this provides a means to correlate topographical information with spectroscopic data.

Several near-field IR microscope designs have been published. ${ }^{9-13}$ To date, however, only two of them ${ }^{12,13}$ have made use of IR-transmitting fibers. This is probably because making NSIM probes from IR fibers has been very difficult.

The primary source of difficulty in making NSIM probes from IR fibers is the physical properties of the fiber material. Perhaps the most tractable set of compounds from which IR-transmitting fibers are made are the chalcogenides. Chalcogenide fibers have good chemical stability and are less brittle than the other families of compounds from which IR fibers have been made. Like optical fibers, they are also glasses, so they can be heat pulled with a capillary puller in a fashion similar to optical fibers. However, heat pulling chalcogenide fibers is significantly more difficult than heat pulling glass $\left(\mathrm{SiO}_{2}\right)$ fibers. It has been accomplished, to our knowledge, only by $\mathrm{Hong}^{13}$ et al. Pulled IR fibers also have relatively low light throughput (on the order of $10^{-6}$, measured in the far field ${ }^{14}$ ). They also have a very long taper length (several mm-see Fig. 1).

Zeisel et al. have reported ${ }^{15,16}$ that chemically etched optical fiber probes have far-field light throughput that is 1001000 times greater than pulled fiber probes, probably because the taper region is much shorter. The longer the taper 


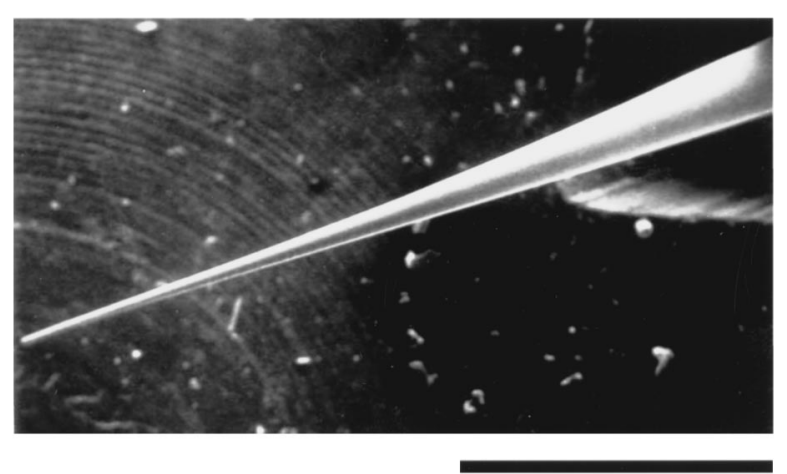

FIG. 1. Scale bar $=1 \mathrm{~mm}$. Scanning electron microscopy micrograph of heat-pulled chalcogenide fiber. Note the long taper length.

region, the longer the region where radiation must propagate in a waveguide narrower than its wavelength. A shorter taper region is thus expected to deliver light more efficiently to the aperture, resulting in higher intensity in both the propagating (far field) and nonpropagating (near-field) components. It is thus a reasonable assumption that the increase in far-field throughput mirrors a proportional increase in near-field intensity.

In the present work we describe the application of a simple chemical etching technique to IR-transmitting chalcogenide fibers. The fiber probes etched this way taper to a sharp point ( $\leqslant 150 \mathrm{~nm}$ end-radius-of-curvature) in approximately one fiber diameter $(\sim 100 \mu \mathrm{m})$. Such sharp probes when scanned in position feedback will easily give spatial topographical resolution of $150 \mathrm{~nm}$. One should distinguish topographical resolution from the spatial resolution of IR imaging, which will be determined by the size of the aperture used.

Simple chemical methods are also described for the removal of the polyamide plastic coating and sulfur-selenide cladding used on these chalcogenide fibers. We also present preliminary data about the IR radiation throughput measured with etched fibers.

\section{MATERIALS AND METHODS}

Chalcogenide fibers were obtained from Amorphous Materials, Inc., 3130 Benton, Garland, TX 75042. Both thin (unclad, but plastic coated) and thick (clad and plastic coated) fibers were used in this study. In both cases the chalcogenide core is an As-Se-Te glass. The cladding is primarily $\mathrm{S}-\mathrm{Se}$. Both types of fiber are Plate No. 94-131-8, Run No. 71395.

Thin fibers have a core diameter of 0.0058 in. $(145 \mu \mathrm{m})$ and a polyamide coating 0.0009 in. $(22.5 \mu \mathrm{m})$ thick [for a total fiber diameter of 0.0076 in. $(190 \mu \mathrm{m})]$.

Thick fibers have a core diameter of 0.010 in. $(250 \mu \mathrm{m})$, $\mathrm{S}-$ Se cladding 0.004 in. $(100 \mu \mathrm{m})$ thick, and a polyamide coating 0.003 in. $(75 \mu \mathrm{m})$ thick [for a total fiber diameter of 0.024 in. $(600 \mu \mathrm{m})]$.

In the process of creating the fiber probes it was useful to use an optical microscope with an epi-fluorescence/ reflectance microscopy attachment. The polyamide coating is slightly fluorescent (under both blue and green light) making

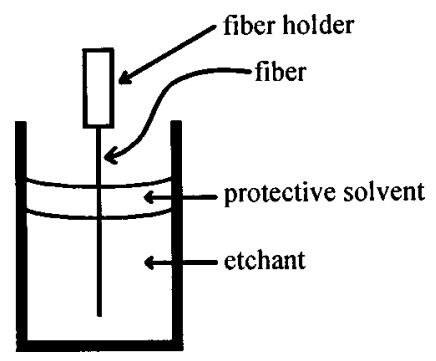

FIG. 2. Schematic of the two-phase etching apparatus. Only 1-2 mm of protective solvent are required. The protective solvent serves to keep the meniscus flat at the fiber-etchant interface.

it easy to tell if it has been completely removed. Microscopy of the probes under both backlighting and reflected light makes it much easier to determine the shape of the fiber.

The best method for examination of the probe tip is scanning electron microscopy. Chalcogenides are semiconductors, so sputtering the tips with gold is not strictly necessary, although it does improve image clarity at very high magnifications.

\section{ETCHING TECHNIQUE}

\section{A. Polyamide coating removal}

The polyamide coating can be easily removed by repeatedly immersing the coated fiber in stirred room-temperature solvent for 1-2 min and wiping with a laboratory tissue. Several solvents work for this purpose, including 4-chloro1-butanol, methanol, and acetone. Properly stripped fibers feel very smooth when wiped with a laboratory tissue; if the polymer has not been completely removed, higher friction can be noticed.

\section{B. Cladding removal}

If the fiber is clad, the cladding must be removed before etching. This can be done by soaking the polyamide-stripped fiber in room-temperature $0.1 \mathrm{M} \mathrm{NaOH}$ for approximately 18 h. At the end of this time, the portion of the fiber that was in the $\mathrm{NaOH}$ solution generally has lost its reflective sheen. Upon wiping gently with a laboratory tissue, the treated portion of the cladding will come off the core.

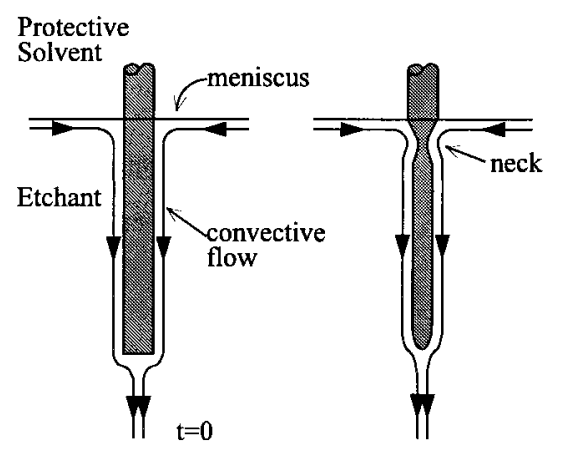

a. b.

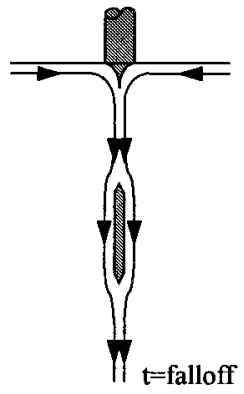

c.
FIG. 3. Schematic of the two-phase etching mechanism under normal conditions (convective control). (a) The system at the beginning of etching, (b) after etching has progressed approximately halfway to completion, and (c) at the falloff point. 


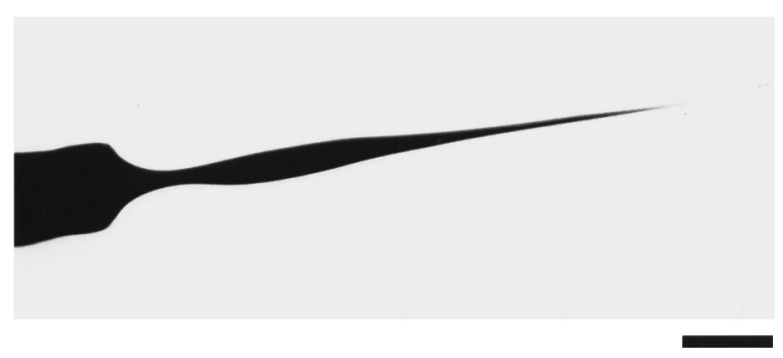

FIG. 4. Scale bar $=150 \mu \mathrm{m}$. Optical micrograph of a fiber removed from the etching solution before the falloff point. Note the pronounced neck.

If the fiber is removed from the $\mathrm{NaOH}$ solution too soon, wiping will not remove the cladding, but the fiber may be safely put back in the solution. We hypothesize that the $\mathrm{NaOH}$ must diffuse completely through the cladding before the cladding will come off. If the fiber is left too long in the $\mathrm{NaOH}$ solution $(>24 \mathrm{~h})$, the $\mathrm{NaOH}$ will very slowly start to etch the chalcogenide core.

If the polyamide comes into contact with the $\mathrm{NaOH}$ solution, the core may become extremely brittle, especially at the point of contact.

\section{Chalcogenide core etching}

Once the core has been exposed, etching the core is straightforward. A container is prepared with a two-phase etching system. The lower phase is the etchant solution, while the upper phase is a protective solvent (see Fig. 2).

The etchant solution is piranha solution, a 7:3 mixture of concentrated sulfuric acid and 30\% hydrogen peroxide. Caution! Piranha solution may react violently with organic compounds and should not be stored in sealed containers. Many different organic compounds may serve as the protective solvent. The authors have had the most success with tetramethylpentadecane (TMPD), polydimethylsiloxane, and $\mathrm{CCl}_{4}$, with TMPD having the best long-term stability against piranha solution.

The fiber is immersed in the etching system. At room temperature and without stirring, the fiber will "neck in" approximately one diameter down from the solvent-piranha meniscus. Ultimately, the neck will be completely etched through, and the lower piece will fall off. (See Figs. 3 and 4.)

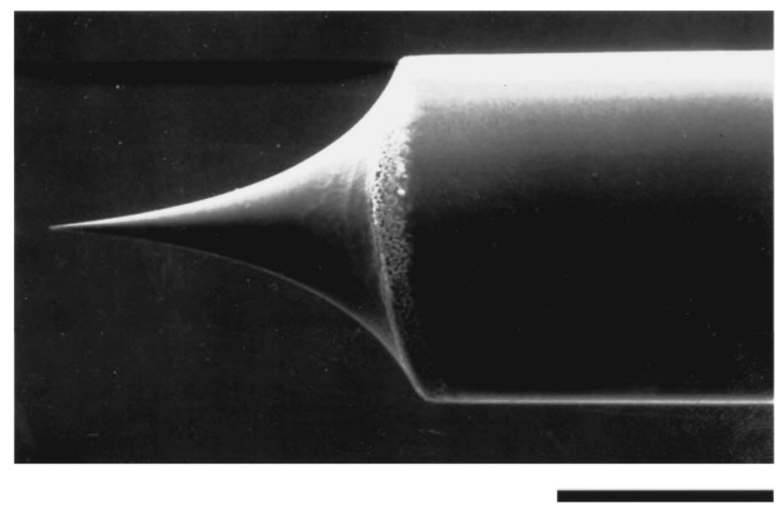

FIG. 5. Scale bar $=100 \mu \mathrm{m}$. Scanning electron microscopy micrograph of a $145 \mu \mathrm{m}$ diameter core chalcogenide fiber etched by the described method.

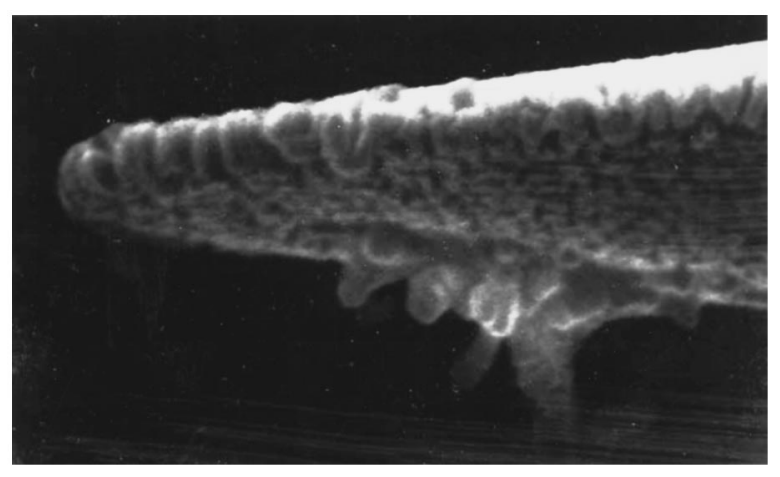

FIG. 6. Scale bar $=1 \mu \mathrm{m}$. Zoom onto the apex of the tip shown in Fig. 5 . The corrugations and the structure on the underside of the tip are believed to be due to the thin layer of gold applied for scanning electron microscopy imaging.

This is referred to as the "falloff" point. The time it takes to reach falloff depends on the age of the piranha solution, its temperature, and the thickness of the chalcogenide core. As a guideline, with freshly made piranha at room temperature, and a $145 \mu \mathrm{m}$ core, etching takes $\sim 15 \mathrm{~min}$. With 2 month old piranha (stored at $5{ }^{\circ} \mathrm{C}$ ), starting slightly below room temperature, and a $250 \mu \mathrm{m}$ core, etching takes $\sim 50 \mathrm{~min}$. Etching times are relatively consistent, but variations of a few minutes must be allowed for.

Approximately $30 \mathrm{~s}$ after the falloff, the fiber is withdrawn from the etchant, washed in gently stirred roomtemperature methanol, and gently blown dry with $\mathrm{N}_{2}$ (blowing on-axis onto the tip, rather than from the side).

Figures 5 and 6 show an etched unclad $145 \mu \mathrm{m}$ fiber. Figures 7,8 , and 9 show an etched, clad fiber with a $250 \mu \mathrm{m}$ core.

\section{Chalcogenide core etching mechanism}

The mechanism of tip formation is believed to be convective control of chemical etching. This etching is hypothesized to occur as follows.

As the etching agent dissolves the fiber, the solution density increases next to the fiber surface. Since it is more dense

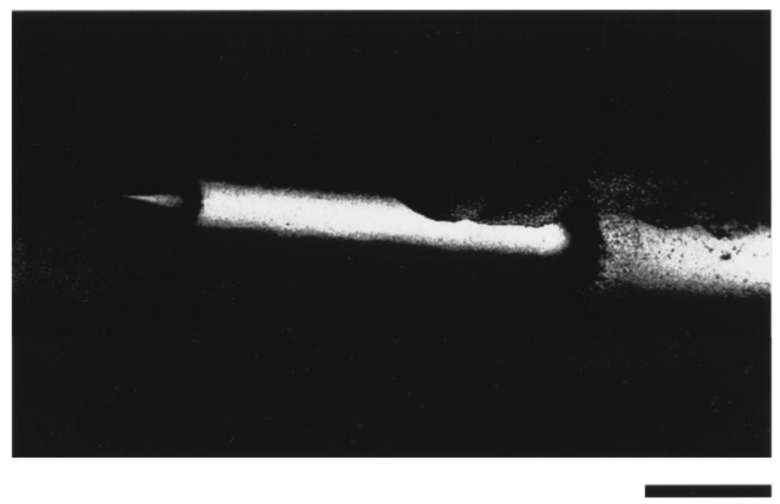

FIG. 7. Scale bar $=500 \mu \mathrm{m}$. Scanning electron microscopy micrograph of a clad $250 \mu \mathrm{m}$ diameter core chalcogenide fiber etched by the described method. Removal of the clad and etching of the core are visible. The "blob" at the top is silver paint applied to provide a conductive path to ground for scanning electron microscopy imaging. 


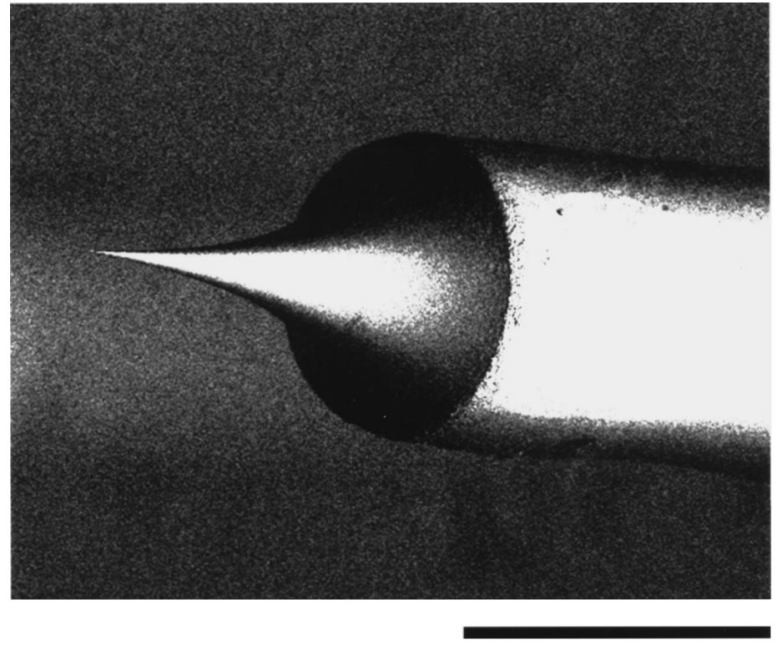

FIG. 8. Scale bar $=250 \mu \mathrm{m}$. Zoom onto the tip of the tip shown in Fig. 7.

than the rest of the solution, it flows down the fiber; under these conditions the flow is laminar. As it flows down the fiber, more etchant solution must move to take its place. Since there is a fluid layer moving parallel to the surface of the fiber everywhere but close to the meniscus (the "top" of where the etchant touches the fiber), new etchant solution enters the convection pattern primarily at the meniscus, as shown in Fig. 3. Since the etchant solution contacting the fiber is more reactive (i.e., contains more $\mathrm{H}_{2} \mathrm{O}_{2}$ and less dissolved chalcogenide) at the meniscus, it etches faster there. This results in a necking effect. Eventually the neck will be dissolved completely away, and the fiber below the neck will fall.

The protective solvent overlayer serves to make tip shape more smooth and reproducible. Fibers etched without solvent overlayers are typically asymmetric and lack the smooth concave conical shape of fibers etched with solvent overlayers. (See Fig. 10.) Experimentally, it is observed that without a solvent overlayer, the meniscus of piranha solution at an inserted fiber curves up, whereas with a solvent overlayer the meniscus of the piranha solution at the fiber is flat. This meniscus curvature is believed to disrupt the convection pattern responsible for producing smooth, concave-conical tips.

According to the convective control hypothesis, if the

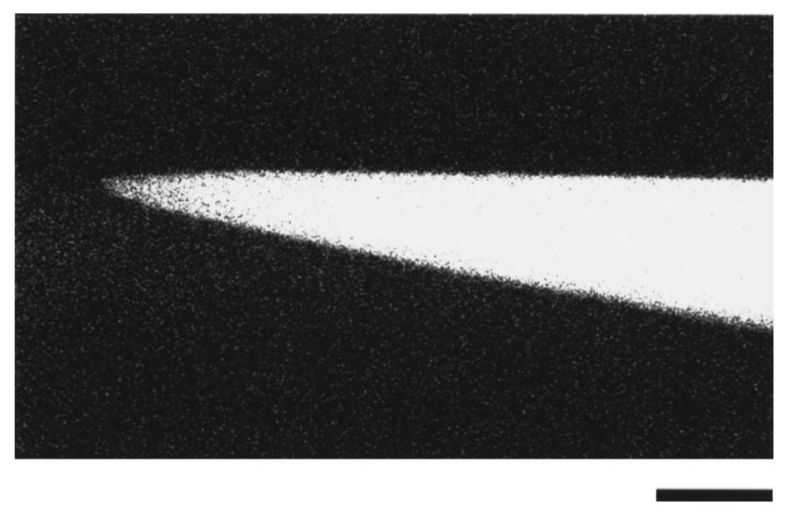

FIG. 9. Scale bar $=1 \mu \mathrm{m}$. Zoom onto the tip apex of the tip shown in Fig. 7.
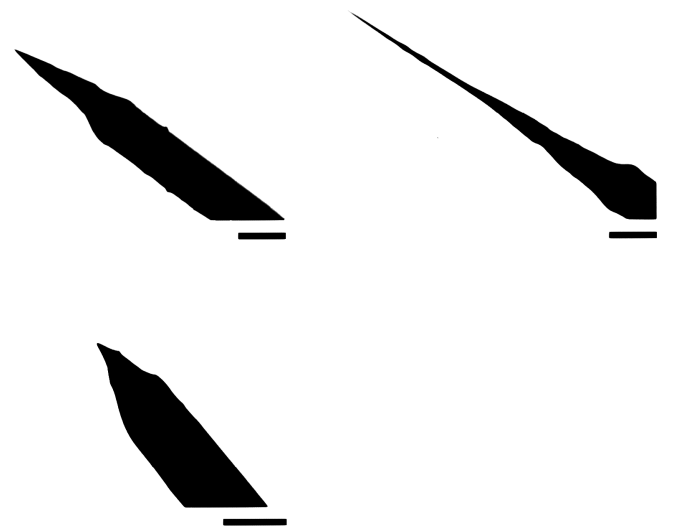

FIG. 10. Scale bars $=150 \mu \mathrm{m}$. Optical micrographs of tips etched without protective solvent overlayers. Note the asymmetry and large-scale corrugation.

effects of convection are reduced relative to diffusion and reaction, more isotropic etching would be expected. (See Fig. 11.) Three different experiments were performed to test this prediction. In the first experiment, etching was performed in the normal apparatus, but at reduced temperature $\left(5^{\circ} \mathrm{C}-\right.$ see Fig. 12). Reduced temperatures increase the viscosity of the piranha solution, thus making convection slower. In the second experiment, etching was performed in the normal apparatus, but with fairly rapid stirring of the piranha solution. Stirring essentially equalizes the concentration of $\mathrm{H}_{2} \mathrm{O}_{2}$ and dissolved chalcogenide throughout the piranha solution (see Fig. 13). In the third experiment, a glass capillary (i.d. $\sim 1.5 \mathrm{~mm}$ ) was used instead of a glass vial (i.d., $\sim 2.5 \mathrm{~cm}$ ). Since the fiber itself took up a substantial portion of the interior space in the capillary, convection was seriously impeded (results not shown). In all three cases, long, high aspect-ratio fiber tips without evidence of necking were produced. It is more difficult to judge "completion" with these fibers, since there is no "falloff" event.

From our hypothesis, we would also expect that the neck should always point straight down from the meniscus. To test this prediction, etching was performed in the normal apparatus, but with the glass vial tilted at $\sim 60^{\circ}$. As predicted, the tip thus produced pointed off-axis by approximately $60^{\circ}$ (results not shown). The tip was, however, asymmetric and not very sharp.

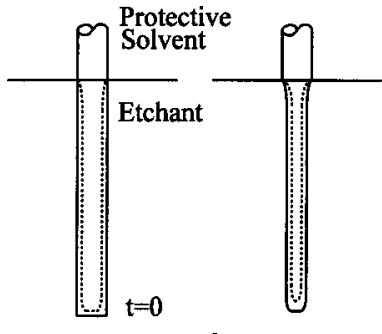

a. b.

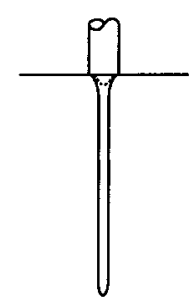

c.

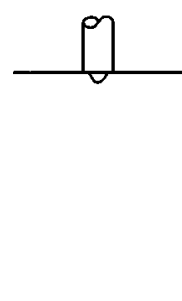

d.
FIG. 11. Schematic of the two-phase etching mechanism under conditions of isotropic etching. The dotted lines qualitatively indicate rates of etching, or the new profile of the tip after a discrete time-interval. No necking or falloff is expected. 


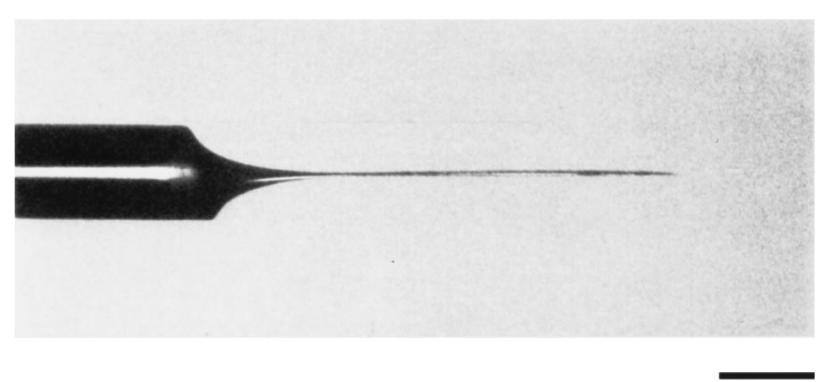

FIG. 12. Scale bar $=150 \mu \mathrm{m}$. Optical micrograph of a $145 \mu \mathrm{m}$ core chalcogenide fiber etched at reduced temperature $\left(5^{\circ} \mathrm{C}\right)$.

\section{IR RADIATION THROUGHPUT MEASUREMENTS}

For transmission measurements chalcogenide glass infrared fibers with a core diameter of $250 \mu \mathrm{m}$ were used. A two-step etching procedure has been used to ensure the straight taper and sharp taper angle. At the first step, the long (about $1 \mathrm{~mm}$ ) concave taper has been achieved during about 30 min of etching, then the fiber was inserted $\sim 0.5 \mathrm{~mm}$ further into the etching solution and allowed to react another 1-2 min, resulting in a straight taper of about $20^{\circ}$ (half angle). The resulting shape of the etched tip is shown in Fig. 14 with optical original magnification $\times 200$. The thickest portion of the fiber in this picture corresponds to initial core diameter of $250 \mu \mathrm{m}$. The second step of etching is clearly seen on this photograph at about $0.6 \mathrm{~mm}$ from the tip.

The etched fiber was then coated with a $190 \mathrm{~nm}$ layer of gold. During the gold deposition the fiber was rotated around its axis oriented at about $45^{\circ}$ to the direction towards the source, with the tip pointing down (towards the evaporation boat). Such a coating geometry should result in complete opacity of the tapered tip.

The coated fiber was then fixed in a micromanipulator on an inverted microscope (Zeiss, Axiovert 35) with the tip pointing down, towards the objective. For detection of the IR radiation the Cassegrain objective has been used with NA 0.65 (Ealing Electrooptics Inc., Holliston, MA). A MCT detector (Kolmar Technologies Inc., Conyers, GA) has been used for detection of the IR radiation collected by the objective and reflected towards the epi-illumination port (the collimator lens has been removed to ensure transmission of the IR radiation). The reflector slider contained two reflector slides-one with gold coating for full reflection of the measured IR light, and the other-an uncoated glass slide-for observation of the fiber tip with epi-illumination. (A Tshaped adapter was used at the rear port of the microscope to

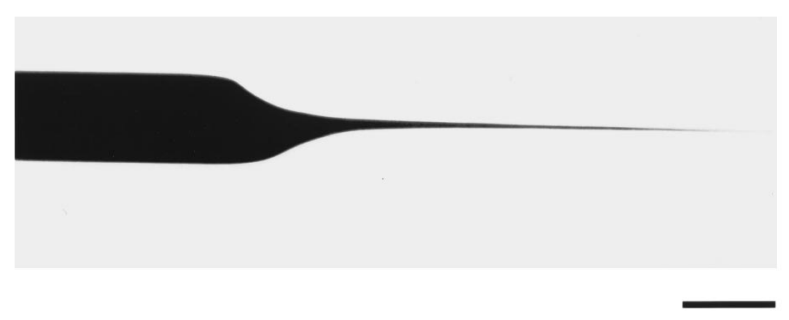

FIG. 13. Scale bar $=150 \mu \mathrm{m}$. Optical micrograph of a $145 \mu \mathrm{m}$ core chalcogenide fiber etched in stirred piranha solution.

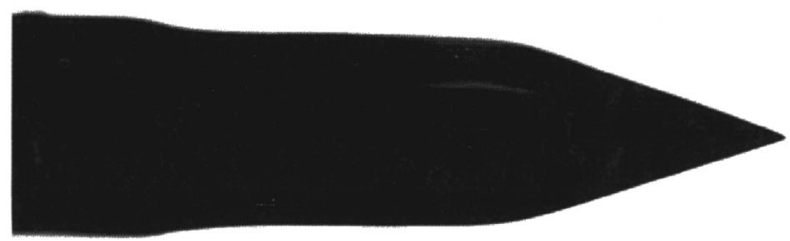

FIG. 14. Optical micrograph of a $250 \mu \mathrm{m}$ core chalcogenide fiber etched in piranha solution utilizing two-step procedure (described in text). The thickest portion of the fiber in this picture corresponds to initial core diameter.

accommodate both the illumination lamp and the MCT detector.)

The radiation of a free electron laser (FEL) was focused on the input end of the fiber (which was about $1 \mathrm{~m} \mathrm{long).}$ Macropulses of about $3 \mathrm{~ms}$ duration and repetition rate of 10 $\mathrm{Hz}$ were applied at the wavelength of $6.01 \mu \mathrm{m}$ with an average power of $2.5 \mathrm{~mW}$. A small portion of the FEL beam was reflected to the reference detector for monitoring the input beam intensity, and the ratio of the signal received through the fiber to the reference signal was recorded to ensure the signal stability at various input energies.

The fiber tip was positioned in the focus of the objective (the tip was observed through the microscope with $\times 20$, $\times 40$, and $\times 100$ refractive objectives under epi-illumination) and the IR signal has been detected using the reflective objective. The nonzero signal at this point indicated leakage of the radiation through the holes in coating. Such holes may result form the dust particles occasionally stack to the fiber prior to coating or from the small droplets of gold ejected from the evaporation boat. After the measurement of the background signal, a $0.1 \mathrm{~mm}$ thick glass slide was positioned under the fiber tip. A thin polishing film with a particle size of $0.05 \mu \mathrm{m}$ was attached to the slide. The microscope objective was focused on the film, and then the fiber was carefully brought into contact with the film which was detected by slight movement of the film out of focus resulting from deflection of the film and/or the glass slide supporting the film. To ensure the flexibility of the system, the slide was held only at one side providing a long cantilever under the polishing film and the film was attached to the slide at one end providing some free space between the film and the glass under the tip. The film was scanned in $x$ and $y$ directions over a few microns, then the tip was withdrawn from contact, and the slide with polishing film was removed.

The resulting flat aperture was observed through the $\times 100$ objective and its dimensions measured with a resolution of about $0.5 \mu \mathrm{m}$. Then the IR signal transmitted through the tip was measured using the reflective objective. The polishing procedure and corresponding transmission measurements have been repeated until the taper have been completely removed, providing the signal for normalization of the measured throughputs. The resulting curve of the throughput (with subtracted background) depicted as a function of the aperture area is shown in Fig. 15(a).

We used the aperture area instead of diameter due to the fact that the aperture was not round and the elliptical axis ratio varied with the distance from the tip. In Fig. 15(b) the power fluence at the tip exit is depicted as a function of the 


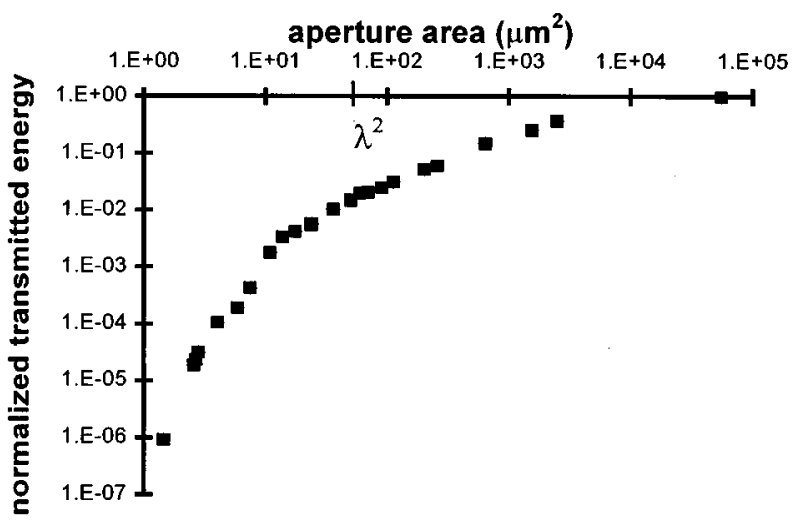

(a)

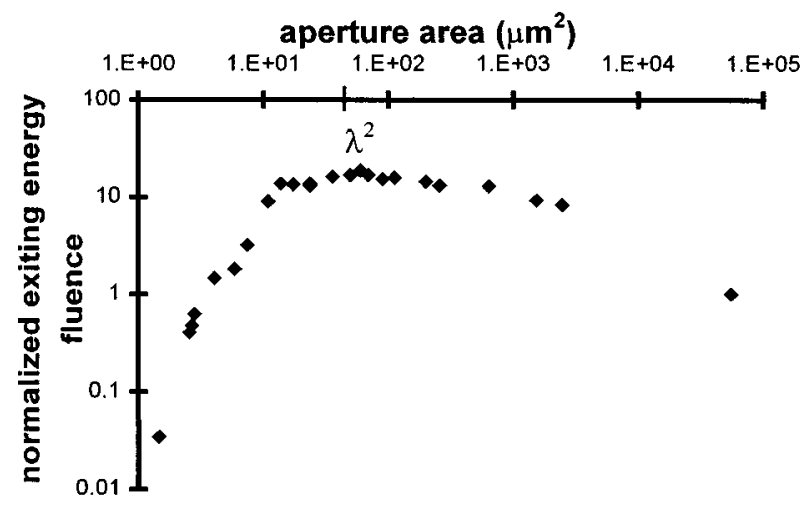

(b)

FIG. 15. IR radiation transmission at $\lambda=6.01 \mu \mathrm{m}$ through an etched and gold coated fiber as a function of exit aperture area; (a) normalized transmission, (b) normalized transmitted power fluence at the surface of exit aperture.

tip size-this curve is a result of division of transmitted power [shown in Fig. 15(a)] on corresponding aperture area. As can be seen on this graph, the power fluence grows at the beginning of the taper up to about 15 times the input fluence due to the light concentration, and remains constant upto the aperture size of about $10 \mu \mathrm{m}^{2}$, which correspond to about $(\lambda / 2)^{2}$ (typical behavior of the macroscopic tapered fibers). At smaller apertures the power fluence drops due to exponential attenuation of the light intensity in the waveguide below the cutoff diameter. ${ }^{17}$ The throughput becomes lower than $10^{-4}$ at the aperture size below about $4 \mu \mathrm{m}^{2}$, which corresponds to the round aperture diameter of about $\lambda / 3$.

The described results indicate higher throughput of etched fiber probes as compared to pulled probes. Demonstrated characteristics of etched probes make it possible to substitute an optical parametric oscillator (OPO) laser as a light source. As OPO lasers are far more common and much less costly than FELs $(\sim \$ 250000$ versus $\$ 1000000$ + shielded facility cost), this would make NSIM a vastly more accessible technique.

Another potential use of etched IR fiber probes is for high-spatial-resolution temperature measurements, such as those required in semiconductor device diagnostics. Tapered IR-transparent fibers are also used as attenuated total reflectance "cells" in Fourier transform infrared spectroscopy (FTIR). ${ }^{18}$ Chemical etching, as opposed to hot-filament pulling, may also be a useful way to fabricate probes for this application.

\section{ACKNOWLEDGMENTS}

We thank Shyamsunder Erramilli, Alan Schwettman, Mi Hong, Andrew Jeung, and Philip Huie for encouragement, for providing the cladded fiber, and for help with testing of etched probes at the Free Electron Laser Facility at Stanford University. D.A.K. was supported by DARPA and the Beckmann Institute. M.A.U. was supported by a fellowship from Lucent Technologies. D.V.P. was supported in part by Office of Naval Research Grant No. N00014-94-1-1024. This support is gratefully acknowledged.

${ }^{1}$ A. Harootunian, E. Betzig, M. Isaacson, and A. Lewis, Appl. Phys. Lett. 49, 674 (1986).

${ }^{2}$ E. Betzig, J. Trautman, T. Harris, J. Weiner, and R. Kostelak, Science 251, 1468 (1991).

${ }^{3}$ D. Courjon and C. Baineier, Rep. Prog. Phys. 57, 989 (1994).

${ }^{4}$ R. Kopelman and W. H. Tan, Appl. Spectrosc. Rev. 29, 39 (1994).

${ }^{5}$ E. Betzig and J. K. Trautman, Science 257, 189 (1992).

${ }^{6}$ J. K. Trautman, J. J. Macklin, L. E. Brus, and E. Betzig, Nature (London) 369, 40 (1994).

${ }^{7}$ Assuming cutoff at $50 \mathrm{~dB} / \mathrm{km}$, or $20 \mathrm{~dB} / \mathrm{m}$.

${ }^{8}$ G. A. Valaskovic, M. Holton, and G. H. Morrison, Appl. Opt. 35, 1215 (1995).

${ }^{9}$ G. A. Massey, J. A. Davis, S. M. Katnik, and E. Omon, Appl. Opt. 24, 1498 (1985)

${ }^{10}$ T. Nakano and S. Kawata, Optik (Stuttgart) 94, 159 (1993).

${ }^{11}$ A. Lahrech, R. Bachelot, P. Gleyzes, and A. C. Boccara, Opt. Lett. 21, 1315 (1996).

${ }^{12}$ A. Piednoir, C. Licoppe, and F. Creuzet, Opt. Commun. 129, 414 (1996).

${ }^{13}$ M. K. Hong, S. Erramilli, P. Huie, G. James, and A. Jeung, Proc. SPIE 2863, 54 (1996).

${ }^{14} \mathrm{~S}$. Erramilli and M. Hong (personal communication).

${ }^{15}$ D. Zeisel, S. Nettesheim, B. Dutoit, and R. Zenobi, Appl. Phys. Lett. 68, 2491 (1996).

${ }^{16}$ D. Zeisel, B. Dutoit, V. Deckert, T. Roth, and R. Zenobi, Anal. Chem. 69, 749 (1997).

${ }^{17}$ For example, T. R. Corle and G. S. Kino, Confocal Scanning Optical Microscopy and Related Imaging Systems (Academic, San Diego, 1996), p. 207.

${ }^{18}$ M. C. Ertan-Iamontagne, S. R. Lowry, W. R. Seitz, and S. A. Tomellini, Appl. Spectrosc. 49, 1170 (1995). 